\title{
LIBYA ANTIQUA
}

The annual review of the Department of Antiquities is the major journal for the publication of reports and articles concerning the archaeology of Libya. Earlier issues can be purchased from:-

L'Erma di Bretschneider, Via Cassiadoro, 19, Rome, Italy.

Prices of the volumes vary but are always reasonable, and all details can be obtained from the distributor in Rome.

\section{Libyan Studies, Select Papers of the late R. G. Goodchild}

(edited by Joyce Reynolds) pp 345, 96 photos, 75 fits

available from The Secretary, Society for Libyan Studies, 31-34, Gordon Square, London, WC1H OPY

$$
\text { Price } £ 5.00+\text { postage }
$$




\section{The Society for Libyan Studies}

President:

Vice-Presidents:

Chairman:

Hon. Treasurer:

Secretary:
Officers and Council 1979-80

*J. B. Ward-Perkins Esq., C.M.G., C.B.E., F.B.A., F.S.A.

Mrs. S. K. Strong

Mrs. O. P. F. Hackett

*Charles Daniels, M.A., F.S.A.

*T. L. Taylor Esq.

*Mrs. S. Lewis, B. Phil., M.A.

\section{Council}

*J. A. Allan Esq., Ph.d., F.R.G.S. (Royal Geographical Society)

G. W. W. Barker Esq., Ph.d., (Prehistoric Society)

J. Boardman Esq., F.B.A. (British School at Athens)

Professor J. D. Evans, Ph.D., F.S.A. (London University, Institute of Archaeology)

G. Fehervari Esq., Ph.D.

Professor W. B. Fisher, Dr. de l'Univ.

Professor G. D. B. Jones, M.A., D.Phil., F.S.A.

P. M. Kenrick Esq.

R. A. Lawless Esq., Ph.D.

*J. H. Little Esq.

*J. A. Lloyd, Esq., Ph.D.

Miss J. M. Reynolds, F.S.A. (British School at Rome)

J. A. Riley Esq., Ph.D.

A. H. K. Slater Esq. (Egypt Exploration Society)

Mrs. S. K. Strong

${ }^{*}$ Members of the Executive Committee 